\title{
Effect of the Carbon Source on Facile Synthesized Si/Graphite Composites and their Electrochemical Performance
}

\author{
Chan Liu ${ }^{l}$, Zhiqiang Gu ${ }^{1 *}$,Zan Zhou ${ }^{1}$,Yuxi Chen ${ }^{l}$, Yuede He ${ }^{1}$, Xiaohong Xia ${ }^{l}$,Hongbo Liu ${ }^{1,2 * *}$ \\ ${ }^{1}$ College of Materials Science and Engineering, Hunan University, Changsha, China \\ ${ }^{2}$ Hunan Province Key Laboratory for Advanced Carbon Materials and Applied Technology, Hunan \\ University, Changsha, Hunan, 410082, China \\ *E-mail: 317295208@qq.com (Zhiqiang Gu); hndxlhb@163.com (Hongbo Liu);
}

doi: $10.20964 / 2019.06 .22$

Received: 7 February 2019 / Accepted: 20 March 2019 / Published: 10 May 2019

\begin{abstract}
Silicon as the most promising candidate of anode for Li-ion batteries has high capacity $\left(4200 \mathrm{mAh} \mathrm{g}^{-1}\right)$, but the poor performance limits its practical application. In this paper, we explore the effects of different carbon sources on silicon/graphite composites. It is found that the sucrose as the carbon source can bind with the silicon particles and graphite well and form a continuous three-dimensional conductive network, resulting in improving the electrochemical properties of the materials. This silicon/graphite composites using sucrose as carbon source exhibit an excellent cyclic performance. The capacity retention is 88.5 $\%$ with a high capacity of $606 \mathrm{mAh} \mathrm{g}^{-1}$ after 50 cycles. Moreover, the effect of sucrose concentration on the electrochemical performance is investigated. When the additive amount of sucrose is $1.0 \mathrm{~g}$, the materials can achieve the best electrochemical performance, which demonstrates that a suitable carbon layer is beneficial to the silicon/graphite composites.
\end{abstract}

Keywords: lithium-ion battery, Si/G composites, anode, carbon sources.

\section{$\underline{\text { FULL TEXT }}$}

(C) 2019 The Authors. Published by ESG (www.electrochemsci.org). This article is an open access article distributed under the terms and conditions of the Creative Commons Attribution license (http://creativecommons.org/licenses/by/4.0/). 